\title{
O Conhecimento do Nome das Letras e a sua Relação com o Desenvolvimento da Escrita: Evidência de Adultos Iletrados
}

\author{
The Relationship between Letter Name Knowledge and the Development \\ of Spelling: Evidence from Illiterate Adults
}

\author{
Marcela Fulanete Corrêa, Cláudia Cardoso-Martins* \& Larissa Assunção Rodrigues \\ Universidade Federal de Minas Gerais
}

\begin{abstract}
Resumo
Dezenove adultos que não haviam começado a ler foram solicitados a escrever uma lista de palavras da melhor maneira que pudessem. De modo geral, suas escritas foram compostas de letras cujo nome podia ser claramente detectado na pronúncia das palavras (e.g., a escrita IU para chinelo). Esses resultados sugerem que adultos iletrados utilizam seu conhecimento do nome das letras para conectar a escrita à fala. Como parece ocorrer entre crianças, o uso dessa estratégia resultou, algumas vezes, em escritas "silábicas", isto é, escritas em que o número de letras corresponde ao número de sílabas na pronúncia da palavra.

Palavras-chave: Adultos iletrados; Escrita; Conhecimento do nome das letras.
\end{abstract}

\begin{abstract}
Nineteen adults who did not know how to read were asked to spell a list of words as well as possible. In general, their spellings consisted of letters whose names could be clearly heard in the pronunciation of the word (e.g., the spelling I and $\mathrm{U}$ for chinelo, in which the names of the letters $i$ and $u$ can be detected in the pronunciation of the word). These results suggest that illiterate adults use their knowledge of letter names to connect print to speech. Similar to what has been observed among preschool children, the use of this strategy resulted, sometimes, in so-called "syllabic" spellings, that is, spellings in which the number of letters correspond to the number of syllables in the pronunciation of the word.

Keywords: Illiterate adults; Spelling; Letter-name knowledge.
\end{abstract}

Os resultados de vários estudos revelam uma associação estreita entre o conhecimento do nome das letras e a habilidade inicial de leitura e escrita em uma ortografia alfabética (e.g., Cardoso-Martins \& Batista, 2005; Pennington \& Lefly, 2001; Pollo, Kessler, \& Treiman, 2005; Treiman, Tincoff, \& Richmond-Welty, 1996). Uma razão óbvia para essa associação diz respeito ao fato de que os nomes das letras são frequentemente escutados na pronúncia das palavras (por exemplo, o nome da letra $t$, /te/, pode ser claramente detectado na pronúncia da palavra telefone). É possível, portanto, que a criança utilize o seu conhecimento do nome das letras para conectar a escrita à fala. Com efeito, Treiman et al. (1996) mostraram que crianças norte-americanas em idade pré-escolar acham mais fácil dizer que uma palavra como beach

\footnotetext{
* Endereço para correspondência: Universidade Federal de Minas Gerais, Faculdade de Filosofia e Ciências Humanas, Departamento de Psicologia, Av. Antônio Carlos, 6627, Campus Pampulha, Belo Horizonte, MG, Brasil, CEP 31270-901.E-mail: cacau@fafich.ufmg.br

Agradecemos aos adultos que participaram do estudo. A preparação desse trabalho foi possível graças a uma bolsa de pesquisa do Conselho Nacional de Desenvolvimento Científico e Tecnológico (CNPq) à segunda autora.
}

(praia), por exemplo, começa com a letra $b$ do que uma palavra como bone (osso). Embora ambas as palavras sejam igualmente frequentes em livros para crianças e comecem com o mesmo som consonantal, o nome da letra $b$ em inglês (/bi/) é claramente audível na pronúncia da palavra beach. Por outro lado, apenas o fonema /b/ no início do nome da letra $b$ pode ser detectado no início da forma oral da palavra bone.

O mesmo fenômeno tem sido observado em línguas tão diversas como o hebraico (Levin, Patel, Kushnir, \& Barad, 2002) e o português do Brasil (Cardoso-Martins \& Batista, 2005; Cardoso-Martins \& Corrêa, 2008; Cardoso-Martins, Corrêa, Lemos, \& Napoleão, 2006; Pollo, Treiman, \& Kessler, 2008). Por exemplo, CardosoMartins e Batista (2005) pediram a crianças em idade pré-escolar para escrever pares de palavras que começavam com a mesma letra e som consonantal (e.g., dedo e dado). Para cada par, o nome da consoante inicial podia ser claramente detectado na forma oral de uma das palavras (e.g., o nome da letra $d$ em dedo), mas não da outra (e.g., dado).

Semelhantemente aos resultados de Treiman et al. (1996), o número de consoantes iniciais representadas corretamente foi maior para as palavras experimentais 
(e.g., dedo) do que para as palavras-controle (e.g., dado) Por outro lado, o número de grafias começando com a primeira vogal na pronúncia da palavra foi maior para as palavras-controle do que para as palavras-experimentais, refletindo o fato de que, ao contrário do que ocorria para essas palavras, o nome da primeira vogal podia ser claramente detectado na pronúncia das palavras-controle (e.g., o nome da vogal $a$ pode ser escutado na pronúncia da palavra dado).

Aparentemente, as crianças se beneficiam do seu conhecimento do nome das letras até mesmo quando apenas parte do nome da letra pode ser detectada na pronúncia da palavra (Levin, et al., 2002; Pollo et al., 2008). Um exemplo particularmente interessante diz respeito ao uso que algumas crianças fazem da letra $h$ no início do desenvolvimento da escrita em português. Embora a letra $h$ não represente som algum em português, Pollo et al. (2008) mostraram que as crianças brasileiras em idade pré-escolar freqüentemente utilizam-na para representar o som /g/, sobretudo quando a seqüência de sons / ga/ pode ser detectada na pronúncia da palavra (e.g., gado, gaveta, etc.). A razão para isso é que, como Pollo et al. (2008) observam, o som /ga/ é claramente audível no nome da letra $h(/ \mathrm{a}$ 'ga/).

A evidência de que as crianças utilizam o seu conhecimento do nome das letras para conectar a escrita à fala tem implicações importantes para uma teoria do desenvolvimento da escrita. Por exemplo, Cardoso-Martins e Batista (2005) sugeriram que tal evidência questiona o modelo de desenvolvimento da escrita de Emilia Ferreiro, sobretudo no que diz respeito à sua interpretação das chamadas escritas silábicas, isto é, escritas em que o número de letras corresponde ao número de sílabas na pronúncia da palavra.

Em uma série de trabalhos, Ferreiro (1989, 1990; Ferreiro \& Teberosky, 1986) tem argumentado que as escritas silábicas constituem a primeira manifestação da compreensão de que a escrita representa os sons da fala. $\mathrm{Na}$ visão de Ferreiro, essas escritas resultam de um longo processo de construção conceitual, mais especificamente, da busca pela criança de um critério que lhe permita controlar as variações no número de letras necessárias para escrever palavras diferentes. Como resultado dessa busca, a criança constrói a hipótese de que as letras na palavra escrita correspondem a segmentos silábicos na palavra oral. Em vista disso, passa a escrever uma e apenas uma letra para cada sílaba na palavra (e.g., a escrita XYS para caballo - cavalo, Ferreiro \& Gómez Palacio, 1982). Como esse exemplo parece demonstrar, o que importa é a correspondência entre o número de letras e o número de sílabas, não a identidade das letras em si.

Ao contrário de Ferreiro, Cardoso-Martins e seus colaboradores (e.g., Cardoso-Martins \& Batista, 2005; Cardoso-Martins \& Corrêa, 2008; Cardoso-Martins et al., 2006) têm argumentado que o conhecimento do nome das letras é um fator importante na gênese das escritas silábicas. Com efeito, as escritas silábicas das crianças em idade pré-escolar que participaram dos estudos mencionados acima eram, em sua maioria, compostas de letras cujo nome podia ser detectado na pronúncia da palavra (e.g., AAA para barata, IIEA para bicicleta, GAIA para gelatina, etc.; ver Cardoso-Martins et al., 2006, p. 638, para outros exemplos semelhantes). Com base nessas observações, Cardoso-Martins e seus colaboradores (Cardoso-Martins \& Batista, 2005; CardosoMartins \& Corrêa, 2008; Cardoso-Martins et al., 2006) têm argumentado que, ao invés de uma construção teórica original, as escritas silábicas resultam da tendência da criança pequena de escrever as letras cujo nome ela é capaz de detectar na pronúncia das palavras. Em português essas letras correspondem frequentemente às vogais. Isso é verdade também para outras línguas em que a escrita silábica tem sido observada como, por exemplo, o espanhol e o italiano (Pontecorvo \& Zucchermaglio, 1990). Uma vez que, por definição, existe apenas uma vogal por sílaba, parece natural que as crianças falantes do espanhol, italiano ou português produzam algumas escritas silábicas no início do desenvolvimento da escrita.

Uma questão interessante diz respeito à generalidade desses resultados. Em outras palavras, a utilização do conhecimento do nome das letras para conectar a escrita à fala é um fenômeno específico do desenvolvimento infantil ou, pelo contrário, é uma estratégia utilizada por pré-leitores em geral, independentemente da sua idade ou nível de desenvolvimento?

O presente estudo investiga essa questão em uma amostra de adultos pré-leitores. Embora vários pesquisadores tenham se interessado pelo processo de alfabetização em adultos com pouca ou nenhuma escolaridade (e.g., Loureiro et al., 2004; Melo, 2006), não temos notícia de nenhum estudo que tenha investigado o papel desempenhado pelo conhecimento do nome das letras nas concepções da escrita de adultos iletrados. No presente estudo, adultos que ainda não sabiam ler foram solicitados a escrever uma lista de palavras da melhor maneira que pudessem. Com base nos resultados descritos anteriormente, esperávamos que a maioria das letras foneticamente apropriadas nas escritas dos adultos correspondesse a nomes de letras na pronúncia das palavras. Esperá-vamos também replicar os resultados encontrados por Cardoso-Martins et al. (2006) sobre a incidência e a natureza das chamadas escritas silábicas entre crianças em idade pré-escolar. Em particular, esperávamos encontrar uma forte associação entre a escrita silábica e o uso do nome das letras para escrever palavras.

\section{Método}

\section{Participantes}

Participaram do estudo 19 adultos (17 mulheres e dois homens) com idade entre 20 e 74 anos $(M=558,55$ meses, $D P=179,84)$. Todos estavam participando de um estudo sobre as estratégias utilizadas por adultos iletrados 
para identificar rótulos ambientais (ver Cardoso-Martins, Rodrigues, \& Ehri, 2003). Apenas adultos que não leram qualquer palavra em um teste de leitura de palavras isoladas foram incluídos no estudo.

Os participantes foram recrutados através de um programa de alfabetização de jovens e adultos de uma cidade pequena localizada no interior do estado de Minas Gerais. Do total de adultos que participaram do estudo, 13 estavam matriculados em classes iniciais de alfabetização. Os demais foram indicados pelos professores e alunos do programa. A maioria das mulheres trabalhava como empregada doméstica em casas de famílias de classe média. Um dos homens era um trabalhador rural aposentado e o outro trabalhava como zelador em uma escola pública.

\section{Materiais e Procedimentos}

Os participantes foram submetidos a várias tarefas, mas apenas aquelas relevantes para o presente estudo são descritas a seguir. Todos os participantes foram avaliados individualmente, em um local reservado no centro de alfabetização ou em seus lares.

Conhecimento do Nome das Letras. Os participantes foram solicitados a nomear 24 letras do alfabeto. As letras apareciam impressas em ordem aleatória em um cartão. Apenas letras maiúsculas foram utilizadas tendo em vista a evidência de que todo material utilizado no programa de alfabetização era impresso em letras maiúsculas.

Reconhecimento dos Sons das Letras. A tarefa do participante consistia em identificar, entre seis letras maiúsculas impressas horizontalmente no centro de um cartão, aquela que correspondia a um som enunciado pelo examinador. A tarefa era composta por um item de treinamento e 15 itens experimentais. Para cada item o experimentador perguntava: "Qual letra faz o som _ ?"

Leitura de Palavras. Os participantes foram solicitados a ler uma lista de 30 palavras encontradas freqüentemente em livros para crianças (Pinheiro \& Keys, 1987). As palavras apareciam impressas em letras maiúsculas, no centro de cartões individuais. Os cartões eram apresentados um de cada vez e pedia-se para os adultos tentar ler a palavra impressa no cartão. Nenhum dos adultos pôde ler qualquer palavra.

Escrita de Palavras. Os participantes foram solicitados a escrever as seguintes 15 palavras: zebu, berço, chinelo, telefone, gaveta, degrau, gemido, chupeta, che$f e$, roça, morcego, voz, jipe, violino e esqueleto. As palavras eram ditadas uma de cada vez pelo examinador e os participantes eram solicitados a escrevê-las em uma folha de papel ou então a produzi-las com a ajuda de letras maiúsculas impressas no centro de cartões individuais. Apenas um participante escreveu as palavras. Todos os demais usaram os cartões para formar as palavras.

Nenhum participante escreveu qualquer palavra corretamente e apenas três escritas, de um total de 285 es- critas possíveis, foram classificadas como alfabéticas (as escritas ROCA e ROSA, ambas para roça, e a escrita GIPI para jipe). Em vista disso, as escritas foram classificadas em dois níveis - pré-alfabético e alfabéticoparcial - que variavam em função da presença ou ausência de letras foneticamente apropriadas, mesmo que convencionalmente incorretas. Uma letra era considerada foneticamente apropriada caso representasse o som em questão em qualquer palavra da língua portuguesa, mesmo que apenas em outros contextos ortográficos (e.g., a letra $c$ na escrita ROCA para roça, foi considerada foneticamente correta, uma vez que ela representa o fonema /s/ em outros contextos como, por exemplo, em cedo e cinto).

As escritas classificadas como pré-alfabéticas não revelavam qualquer compreensão de que as letras representam sons da fala (e.g., RZQ para telefone e TCT para $z e b u$ ). Recusas em escrever também foram classificadas nesse nível. As escritas alfabético-parciais, por outro lado, revelavam um início daquela compreensão. Uma variedade de escritas foi classificada nesse nível. De uma maneira geral, essas escritas não representavam mais do que a metade dos sons contidos na pronúncia da palavra (e.g., Z para zebu, BA para berço, VIOI para violino, GI para gemido, XLO para chinelo, TEI para telefone, etc.), embora algumas representassem um número maior de sons de maneira apropriada (e.g., MOCECO para morcego, GMIDU para gemido, GPI, para jipe, ZEU para $z e b u$, XEI para chefe, HAVTA para gaveta, etc.). Todas as escritas foram codificadas por dois juízes independentes. Em casos de desacordo, os juízes discutiam até chegarem a um consenso.

\section{Resultados}

\section{O Uso do Nome das Letras para Escrever Palavras}

Os adultos revelaram um conhecimento razoável das letras do alfabeto. A proporção média de respostas corretas nos testes de conhecimento do nome e dos sons das letras foi $0,62(D P=0,27)$ e $0,56(D P=0,27)$, respectivamente. Não é surpreendente, portanto, que a imensa maioria das escritas tenha sido classificada como alfabético-parcial $(M=11 ; D P=3,28)$.

Duas análises foram realizadas para avaliar a hipótese de que adultos iletrados utilizam o seu conhecimento do nome das letras para conectar a escrita à fala. Em primeiro lugar, calculamos a proporção de letras foneticamente apropriadas que correspondiam a nomes de letras na pronúncia das palavras (e.g., as letras $z$ e $u$ na escrita ZU para $z e b u$ ) e a proporção que correspondia a fonemas na pronúncia da palavra (e.g., a letra $v$ na escrita VIOI para violino que corresponde ao fonema / $/ \mathrm{l}$ no início da palavra). Algumas vezes, parte do nome da letra podia ser detectado na pronúncia da palavra. Esse era o caso dos segmentos /ga/ e / fi/, os quais podem ser escutados no nome das letras $h$ (/a'ga/) e $x(/ f$ is/), respectivamen- 
te, e que apareceram na pronúncia de algumas das palavras, por exemplo, gaveta e chinelo. Essas consoantes foram classificadas junto com as letras que correspondiam a nomes de letras na pronúncia da palavra. Naturalmente, apenas as escritas alfabético-parciais foram incluídas nessa análise.

Em média, 75\% das letras nas escritas alfabéticoparciais eram letras foneticamente apropriadas. Conforme poderia ser esperado com base na hipótese de que os adultos utilizavam o seu conhecimento do nome das letras para conectar a fala à escrita, a proporção de letras que correspondia a nomes ou partes de nomes de letras $(M=0,63)$ foi significativamente maior do que a proporção que correspondia a fonemas $(M=0,36)$ na pronúncia da palavras, $t_{(18)}=3,32, p<0,01$.

Em uma análise adicional, apenas as consoantes iniciais foram levadas em consideração. Em sete das 15 palavras ditadas - zebu, berço, telefone, degrau, gemido, chinelo e gaveta - o nome ou parte do nome da consoante podia ser detectado no início da pronúncia da palavra. Por outro lado, as consoantes iniciais nas palavras chupeta, chefe, roça, morcego, voz, jipe e violino, não correspondiam a nomes de letras ou parte de nomes de letras, mas sim a fonemas contidos no nome das letras. O número de consoantes foneticamente apropriadas representadas nas escritas dos adultos foi significativamente maior para as palavras do primeiro grupo $(M=5,21, D P=1,62)$ do que para as palavras do segundo grupo $\left(M=2,68, D P=2,03, t_{(18)}=6,203, p\right.$ $<0,001)$. Por outro lado, semelhantemente aos resultados obtidos por Cardoso-Martins e Batista (2005) entre crianças em idade pré-escolar, o número de letras iniciais que representavam a primeira vogal na pronúncia da palavra foi significativamente maior para as palavras do segundo grupo $(M=1,37, D P=0,24)$ do que para aquelas do primeiro $(M=0,37, D P=0,42), t_{(18)}=$ $2,854, p<0,05)$.

\section{Incidência e Natureza das Escritas Silábicas}

Os adultos apresentaram, em média, apenas 5,37 escritas silábicas $(D P=3,18)$, isto é, escritas em que o número de letras correspondia ao número de sílabas nas palavras. Com o objetivo de avaliar a natureza das escritas silábicas, classificamos os adultos como silábicos ou não. Um adulto foi considerado silábico quando oito ou mais de suas escritas haviam sido classificadas como silábicas. Esse procedimento foi utilizado para evitar a possibilidade de que as escritas silábicas tivessem sido produzidas meramente por acaso. Cinco participantes atingiram esse critério. A grande maioria das letras (81\%, em média) nas suas escritas silábicas eram letras foneticamente apropriadas. Além disso, em média, 70\% dessas letras correspondiam a letras cujo nome ou parte do nome podia ser claramente escutado na pronúncia das palavras. De fato, em um número grande de escritas, o nome ou parte do nome de todas as letras podia ser de- tectado na pronúncia da palavra (e.g., EI para chefe, UPA para chupeta, GIU para gemido, ZU para zebu, OA para roça, BU para berço, HVA para gaveta).

\section{Discussão}

O objetivo do presente estudo consistiu em: (a) verificar em que medida adultos iletrados beneficiam-se do seu conhecimento do nome das letras para conectar a escrita à fala; e (b) investigar a incidência e a natureza das escritas silábicas entre adultos iletrados. Os resultados mostraram que, assim como crianças em idade pré-escolar (e.g., Cardoso-Martins \& Batista, 2005; Levin et al., 2002; Treiman et al., 1996), adultos iletrados baseiam-se no seu conhecimento do nome das letras para conectar a escrita à fala. Com efeito, a maioria das letras nas escritas dos adultos que participaram do presente estudo correspondia a letras cujo nome podia ser, total ou parcialmente, escutado na pronúncia da palavra.

Os resultados são também condizentes com os resultados de estudos recentes que investigaram a incidência e a natureza das chamadas escritas silábicas entre crianças em idade pré-escolar e início dos anos escolares (e.g., Cardoso-Martins et al., 2006; Pollo, 2008). Como nesses estudos, o número de escritas silábicas entre os adultos que participaram do presente estudo foi relativamente pequeno. Além disso, conforme descrevemos anteriormente, a maioria das escritas silábicas dos adultos classificados como silábicos era composta de letras cujo nome podia ser claramente detectado na pronúncia da palavra.

Como Cardoso-Martins et al. (2006) observaram em relação à escrita silábica de crianças em idade pré-escolar, os resultados do presente estudo sugerem que, ao invés de uma construção original, a escrita silábica de adultos pré-leitores resulta da sua compreensão de que a escrita representa a fala, e da sua tentativa de representar os sons que é capaz de detectar na pronúncia da palavra por letras foneticamente apropriadas. Nesse sentido, a escrita silábica é mais apropriadamente classificada como uma escrita alfabético-parcial.

Uma limitação do presente estudo diz respeito ao número relativamente pequeno de participantes. Por outro lado, ao que tudo indica, trata-se do primeiro estudo a investigar a relação entre o conhecimento do nome das letras e o desenvolvimento inicial da escrita em adultos pré-letrados. A julgar pelos seus resultados, o uso do nome da letra para conectar a escrita à fala não é um fenômeno específico do desenvolvimento infantil, mas uma estratégia utilizada por pré-leitores em geral, independentemente da sua idade ou nível de desenvolvimento. Como parece ser o caso entre crianças em idade pré-escolar, as letras nas escritas dos adultos iletrados que participaram do presente estudo consistiam, de uma maneira geral, de letras cujo nome podia ser detectado na pronúncia das palavras. Esses resultados sugerem que a escrita de adul- 
tos pré-leitores pode ser descrita em termos das mesmas características que têm sido utilizadas para descrever o desenvolvimento da escrita de crianças que ainda não começaram a ler (e.g., Cardoso-Martins et al., 2006; Ehri, 1998). Uma questão importante para a pesquisa futura consiste em investigar se o mesmo também é verdade para as fases mais avançadas do desenvolvimento da escrita.

\section{Referências}

Cardoso-Martins, C., \& Batista, A.C. E. (2005). O conhecimento do nome das letras e o desenvolvimento da escrita: Evidência de crianças falantes do português. Psicologia: Reflexão \& Crítica, 18(3), 330-336.

Cardoso-Martins, C., \& Corrêa, M. F. (2008). O desenvolvimento da escrita nos anos pré-escolares: Questões acerca do estágio silábico. Psicologia: Teoria e Pesquisa, 24, 279-286.

Cardoso-Martins, C., Corrêa, M. F., Lemos, L. S., \& Napoleão, R. F. (2006). Is there a syllabic stage in spelling stage? Evidence from Portuguese-speaking children. Journal of Educational Psychology, 98, 628-641.

Cardoso-Martins, C., Rodrigues, L. A., \& Ehri, L. C. (2003). Place of environmental print in reading development: Evidence from nonliterate adults. Scientific Studies of Reading, 7, 335-355.

Ehri, L. (1998). Learning to read and learning to spell are one and the same, almost. In C. Perfetti, L. Rieben, \& M. Fayol (Eds.), Learning to spell: Research, theory, and practice across languages (pp. 237-269). Mahwah, NJ: Erlbaum.

Ferreiro, E. (1989). Reflexões sobre a alfabetização. São Paulo, SP: Cortez.

Ferreiro, E. (1990). Literacy development: Psychogenesis. In Y. M. Goodman (Ed.), How children construct literacy: Piagetian perspectives (pp. 12-25). Newark, DE: International Reading Association.

Ferreiro, E., \& Gomez Palacio, M. (1982). Analisis de las perturbaciones en el proceso de aprendizaje escolar de la lectura y de la escritura. Mexico, DF: Siglo XXI.

Ferreiro, E., \& Teberosky, A. (1986). Psicogênese da língua escrita. São Paulo, SP: Artes Médicas.

Levin, I., Patel, S., Kushnir, T., \& Barad, N. (2002). Letter names: Effect on letter saying, spelling, and word recognition in Hebrew. Applied Psycholinguistics, 23, 269-300.

Loureiro, C. S., Braga, L. W., Souza, L. N., Nunes, G., Filho, Queiroz, E., \& Dellatolas, G. (2004). Degree of illiteracy and phonological and metaphonological skills in unschooled adults. Brain and Language, 89, 499-502.

Melo, R. B. (2006). A relação entre consciência fonológica e aquisição da leitura e da escrita de jovens e adultos. Tese de Doutorado não-publicada, Universidade Federal do Rio de Janeiro, RJ.

Pennington, B. F., \& Lefly, D. L. (2001). Early reading development in children at family risk for dyslexia. Child Development, 72, 816-833.

Pinheiro, A. M. V., \& Keys, K. (1987). A word frequency count in Brazilian Portuguese. Unpublised manuscript, University of Dundee, UK.

Pollo, T. (2008). The nature of young children's phonological and nonphonological spellings. Unpublised doctoral dissertation, Washington University, Saint Louis, MO.
Pollo, T. C., Kessler, B., \& Treiman, R. (2005). Vowels, syllables, and letter names: Differences between young children's spelling in English and Portuguese. Journal of Experimental Child Psychology, 92, 161-181.

Pollo, T., Treinan, R., \& Kessler, B. (2008). Preschoolers use partial letter names to select spellings: Evidence from Portuguese. Applied Psycholinguistics, 29, 1-18.

Pontecorvo, C., \& Zucchermaglio, C. (1990). A passage to literacy: Learning in a social context. In Y. M. Goodman (Ed.), How children construct literacy: Piagetian perspectives (pp. 59-98). Newark, DE: International Reading Association.

Treiman, R., Tincoff, R., \& Richmond-Welty, D. (1996). Letter names help children connect print and speech. Developmental Psychology, 32, 505-514. 